\title{
Gamma-ray emission from PSR J0007+7303 using 7 years of Fermi Large Area Telescope observations
}

\author{
Jian $\operatorname{Li}^{1}$, Diego F. Torres ${ }^{1,2}$, Emma de Oña Wilhelmi ${ }^{1}$, \\ Nanda Rea ${ }^{1,3}$, Jonatan Martin ${ }^{1}$
}

\begin{abstract}
Based on more than seven years of Fermi Large Area Telescope (LAT) Pass 8 data, we report on a detailed analysis of the bright gamma-ray pulsar (PSR) J0007+7303. We confirm that PSR J0007+7303 is significantly detected as a point source also during the off-peak phases with a TS value of $262(\sim 16 \sigma)$. In the description of PSR J0007+7303 off-peak spectrum, a power law with an exponential cutoff at $2.7 \pm 1.2 \pm 1.3 \mathrm{GeV}$ (the first/second uncertainties correspond to statistical/systematic errors) is preferred over a single power law at a level of $3.5 \sigma$. The possible existence of a cutoff hints at a magnetospheric origin of the emission. In addition, no extended gamma-ray emission is detected compatible with either the supernova remnant (CTA 1) or the very high energy (>100 GeV) pulsar wind nebula. A flux upper limit of $6.5 \times 10^{-12} \mathrm{erg} \mathrm{cm}^{-2} \mathrm{~s}^{-1}$ in the 10-300 $\mathrm{GeV}$ energy range is reported, for an extended source assuming the morphology of the VERITAS detection. During on-peak phases, a sub-exponential cutoff is significantly preferred $(\sim 11 \sigma)$ for representing the spectral energy distribution, both in the phase-averaged and in the phase-resolved spectra. Three glitches are detected during the observation period and we found no flux variability at the time of the glitches or in the long-term behavior. We also report the discovery of a previously unknown gamma-ray source in the vicinity of PSR J0007+7303, Fermi J0020+7328, which we associate with the $z=1.781$ quasar S5 0016+73. A concurrent analysis of this source is needed to correctly characterize the behavior of CTA 1 and it is also presented in the paper.
\end{abstract}

Subject headings: gamma rays: stars - pulsars: individual: PSR J0007+7303, S5 0016+73. - supernovae: individual (G119.5+10.2)

\footnotetext{
${ }^{1}$ Institute of Space Sciences (CSIC-IEEC), Campus UAB, Carrer de Magrans s/n, 08193 Barcelona, Spain

${ }^{2}$ Institució Catalana de Recerca i Estudis Avançats (ICREA), E-08010 Barcelona, Spain

${ }^{3}$ Astronomical Institute "Anton Pannekoek", University of Amsterdam, Postbus 94249, NL-1090-GE Amsterdam, The Netherlands
} 


\section{INTRODUCTION}

PSR J0007+7303 is a $\sim 316$ ms gamma-ray pulsar discovered by the Fermi Large Area Telescope (LAT) in a blind search (Abdo et al. 2008). Using the timing ephemeris from the LAT, X-ray pulsations from PSR J0007+7303 were detected by XMM-Newton (Lin et al. 2010; Caraveo et al. 2010). Deep searches for optical and radio counterparts of PSR J0007+7303 revealed none (Halpern et al. 2004; Mignani et al. 2013), leading to the characterization of PSR J0007+7303 as a radio-quiet gamma-ray pulsar similar to Geminga (Bertsch et al. 1992) and PSR J1836+5925 (Halpern, Camilo \& Gotthelf 2007; Abdo et al. 2010; Lin et al. 2014).

PSR J0007+7303 is one of the brightest pulsars in The Second Fermi Large Area Telescope Catalog of Gamma-Ray Pulsars (Abdo et al. 2013, 2PC hereafter), providing enough statistics to investigate spectral and timing features and flux variability in detail. PSR J0007+7303 is associated with the composite supernova remnant CTA 1 (SNR; G119.5+10.2), discovered by Harris \& Roberts (1960). CTA 1 possesses a large radio shell that is incomplete towards the north-west (Pineault et al. 1993). ASCA and ROSAT observations revealed a central filled SNR with emission extending to the radio shell (Seward et al. 1995). Chandra observations resulted in the detection of a pulsar wind nebula (PWN) and a jet-like structure (Halpern et al. 2004). The age of CTA 1 is estimated to be around 10 kyr (Pineault et al 1993; Slane et al. 1997; 2004) and the distance is estimated to be 1.4 $\pm 0.3 \mathrm{kpc}$ based on the associated H i shell (Pineault 1997).

The CTA 1 complex was established as an extended gamma-ray source above $500 \mathrm{GeV}$ by VERITAS (Aliu et al. 2013). The extended morphology detected by VERITAS was approximated by a two-dimensional Gaussian of semi-major (semi-minor) axis of $0.30 \pm 0.03$ (0.24 $\left.\pm 0^{\circ} .03\right)$. The TeV photon origin was proposed to be the PWN associated with PSR J0007+7303 (Aliu et al. 2013). With two years of Fermi-LAT observations, the off-peak emission of PSR J0007+7303 appeared to be extended and the morphology was fitted with

a disk of radius $0.7 \pm 0^{\circ} 3$ at $95 \%$ confidence level. Given the extension and spectral shape derived with the two-year statistics, the emission was proposed to be associated with CTA 1 (Abdo et al. 2012).

In this paper, we report further analysis of PSR J0007+7303 and its related SNR CTA 1 using more than seven years of Fermi-LAT data and the newest response functions. 


\section{OBSERVATIONS}

The Fermi-LAT data used for this paper covers 88 months, from 2008 August 4 (MJD 54682 ) to 2015 December 14 (MJD 57370), greatly extending the two years of data coverage reported in Abdo et al. (2012) and the three years of coverage of the 2PC. The LAT is described in Atwood et al. (2009). The analysis of Fermi-LAT data was performed using the Fermi Science Tool\{! 10-00-05 release. Events from the "Pass 8" event class were selected. The "P8R2 V6 Clean" instrument response functions (IRFs) were used in the analysis. All gamma-ray photons within an energy range of $0.1-300 \mathrm{GeV}$ and a circular region of interest (ROI) of $10^{\circ}$ radius centered on PSR J0007+7303 were considered. To reject contaminating gamma rays from the Earth's limb, we selected events with a zenith angle $<90^{\circ}$.

The spectral results presented in this work were calculated by performing a binned maximum likelihood fit (30 bins in the $0.1-300 \mathrm{GeV}$ range) using the Science Tool gtlike. The spectral-spatial model constructed to perform the likelihood analysis includes Galactic and isotropic diffuse emission components ("gll_iem_v06.fits", Acero et al. 2016, and "iso_P8R2_CLEAN_V6_v06.txt", respectively ${ }^{2}$ ) as well as known gamma-ray sources within $15^{\circ}$ of PSR J0007+7303, included in the Fermi LAT Third Source Catalog (Acero et al. 2015, 3FGL hereafter). The spectral parameters and positions were fixed to the catalog values, except for the sources within $3^{\circ}$ of our target. For these latter sources, the spectral parameters were left free. In the phased analysis, the prefactor parameters were scaled to the relative width of the phase interval. The test statistic (TS) was employed to evaluate the significance of the gamma-ray fluxes coming from the sources. The Test Statistic is defined as $\mathrm{TS}=-2 \ln \left(L_{\max , 0} / L_{\max , 1}\right)$, where $L_{\max , 0}$ is the maximum likelihood value for a model without an additional source (the "null hypothesis") and $L_{\max , 1}$ is the maximum likelihood value for a model with the additional source at a specified location. The larger the value of TS, the less likely the null hypothesis is correct (i.e., a significant gamma-ray excess lies on the tested position) and the square root of the TS is approximately equal to the detection significance of a given source.

To search for the possible extension of PSR J0007+7303 in the off-peak gamma-ray emission, we followed the method of Lande et al. (2012). The source is assumed to be spatially extended with a symmetric disk model and we fitted its position and extension with the Pointlike analysis package (Kerr 2010). The extension significance was defined as $\mathrm{TS}_{\text {ext }}=2\left(\ln L_{\text {disk }}-\ln L_{\text {point }}\right)$, in which $L_{\text {disk }}$ and $L_{\text {point }}$ were the gtlike global likelihood of the

\footnotetext{
${ }^{1}$ http://fermi.gsfc.nasa.gov/ssc/

${ }^{2}$ http://fermi.gsfc.nasa.gov/ssc/data/access/lat/BackgroundModels.html
} 
point source and the extended source hypotheses, respectively. The TS maps (i.e., maps of the value of TS for trial positions of an additional source) in this paper are produced with Pointlike.

The systematic errors have been estimated similarly to other Fermi-LAT reports, by repeating the analysis using modified IRFs that bracket the effective area (Ackermann et al. 2012), and artificially changing the normalization of the Galactic diffuse model by $\pm 6 \%$ (2PC). The energy dispersion is not considered in the data analysis, which may be important below $100 \mathrm{MeV}$ but not expected to produce significant changes in the $100 \mathrm{MeV}-300 \mathrm{GeV}$ energy range considered here.

For the Swift/XRT data included in our analysis, we selected Photon Counting (PC) mode $\AA^{3}$ data with event grades 0-12 (Burrows et al. 2005). Source events were accumulated within a circular region with a radius of 30 pixels $(1$ pixel $=2.36$ arcsec $)$. Background events were accumulated within a circular, source-free region with a radius of 60 pixels. Exposure maps were generated with the task XRTEXPOMAP. Ancillary response files were generated with XRTMKARF, which accounts for different extraction regions, vignetting, and pointspread function (PSF) corrections. We analyzed the Swift/XRT 0.3-10 keV data using HEAsoft version 6.14

\section{Off-peak and on-peak phase selection}

We selected photons from PSR J0007+7303 within a radius of 1.2 and a minimum energy of $200 \mathrm{MeV}$, which maximized the H-test statistics (de Jager et al. 1989; de Jager \& Büsching 2010). Adopting the most current ephemeris for PSR J0007+7303 which includes three glitches (M. Kerr 2016, private communication, Table 1), we assigned pulsar rotational phases to each gamma-ray photon that passed the selection criteria, using Tempo2 (Hobbs et al. 2006) with the Fermi plug-in (Ray et al. 2011). The details of the timing analysis and the full timing parameters will be published in the future (M. Kerr et al. 2016, in preparation) $5^{5}$ The phase reference in our ephemeris is the same as in the $2 \mathrm{PC}$, so that the

\footnotetext{
${ }^{3}$ https://www.swift.psu.edu/xrt/software.html

${ }^{4}$ http: //heasarc.nasa.gov/lheasoft/
}

${ }^{5}$ The timing model will be made available as usual from http://fermi.gsfc.nasa.gov/ssc/data/ access/lat/ephems/. 

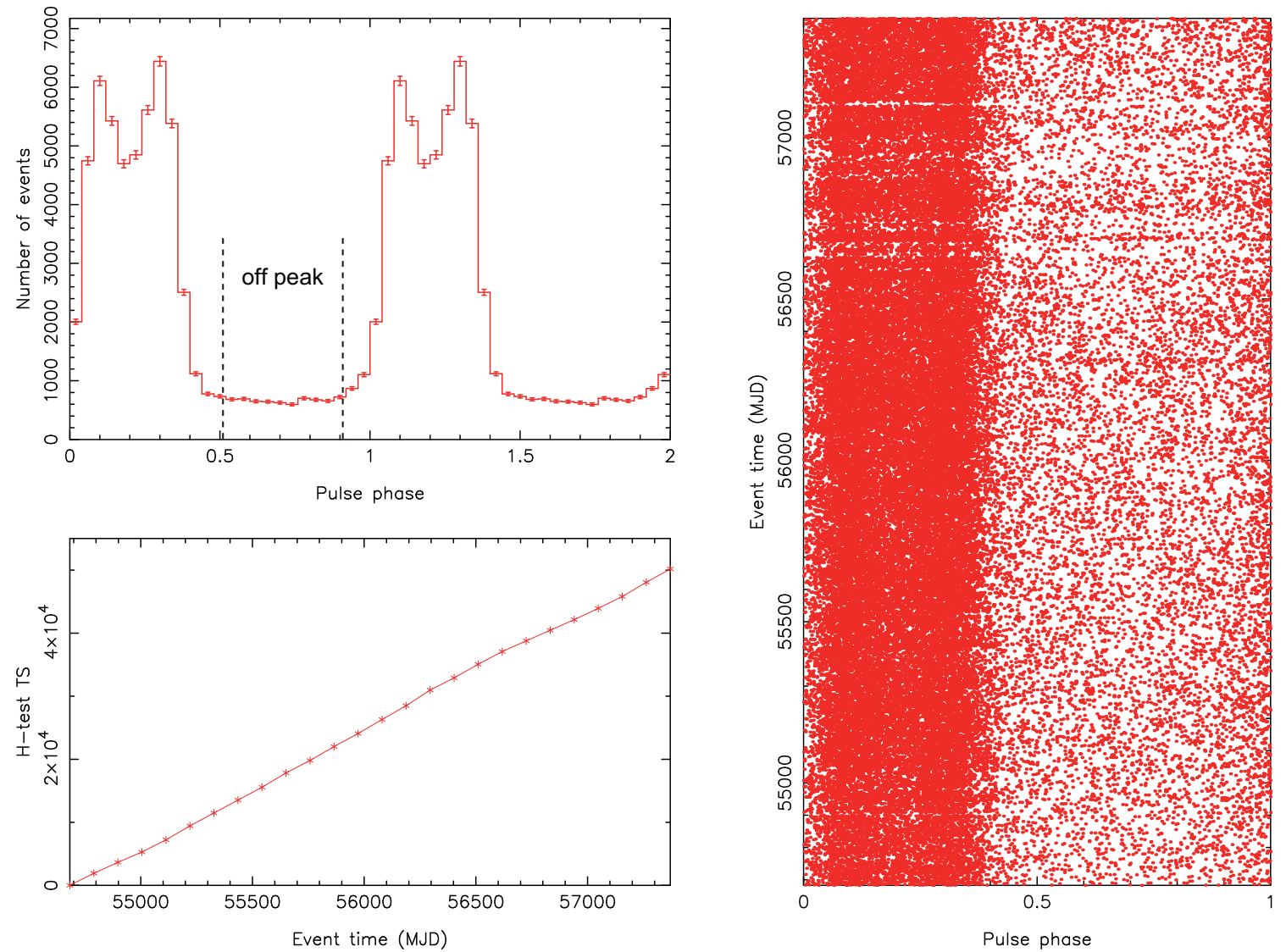

Fig. 1. - PSR J0007+7303 timing results from Tempo2 with the Fermi plug-in. Top-left panel: phase histogram of the analyzed gamma-ray data. Two full rotations are shown for clarity. The off-peak phase interval as determined from a Bayesian block analysis is $\phi=0.511-0.909$ (the region indicated by the black dashed lines) while the on-peak phase interval is $\phi=0.0-0.511$ and $\phi=0.909-1.0$. Bottom-left panel: H-test significance (TS) as a function of time. Right panel: pulse phase for each gamma-ray event versus time. 
profiles could be compared directly. We adopted the Chandra position from Halpern et al. (2004) since the timing noise of PSR J0007+7303 leads to a much lower precision for the measured gamma-ray position (Kerr et al. 2015). The large timing noise also does not allow a reasonable measurement of the proper motion and braking index of PSR J0007+7303. The timing results are shown in Figure 1. The H-test TS increases linearly with time, indicating that the timing ephemeris is valid for the entire data coverage.

We divided the pulse phase of PSR J0007+7303 into two parts, off-peak and on-peak intervals. We begin by deconstructing the pulsed light curve into simple Bayesian Blocks using the same algorithm described in the 2PC, by Jackson et al. (2005) and Scargle et al. (2013). To produce Bayesian Blocks on the pulsation light curve, we extended the data over three rotations, by copying and shifting the observed phases to cover the phase range from -1 to 2 . We define the final blocks to be between phases 0 and 1 . The lowest Bayesian Block is defined as the off-peak phase. To avoid potential contamination from the trailing and/or leading edge of the peaks, we reduce the extent of the block by $10 \%$ on either side, referenced to the center of the block. The off-peak phase is located between $\phi=0.511-0.909$, and is consistent with the off-peak definition for PSR J0007+7303 in the 2PC. The on-peak phase is located at $\phi=0.0-0.511$ and $\phi=0.909-1.0$. In Figure 2, the bottom panel shows the Bayesian block decomposition and the off-peak phase range. More discussion of Figure 2 is presented in Section 6 .

\section{Off-peak analysis}

\subsection{Discovery and analysis of Fermi J0020+7328}

Figure 3 (left panel) shows a TS map of the off-peak phase of PSR J0007+7303. In the vicinity of PSR J0007+7303 there is a previously unknown gamma-ray source. Applying Pointlike, the best-fit position of this gamma-ray source above $100 \mathrm{MeV}$ is $\mathrm{RA}_{J 2000}=4.973$ and $\operatorname{Dec}_{J 2000}=73.462$, with a $95 \%$ confidence error circle radius of 0.044 . By using the fitted position and assuming a power law spectral shape $\left(d N / d E=N_{0}\left(E / E_{0}\right)^{-\Gamma} \mathrm{cm}^{-2} \mathrm{~s}^{-1} \mathrm{GeV}^{-1}\right)$, the gtlike analysis of this gamma-ray source resulted in a TS value of 315 (we shall refer to

this source as Fermi J0020+7328 hereafter). The TS value of PSR J0007+7303 is 281 in the off-peak phases. 


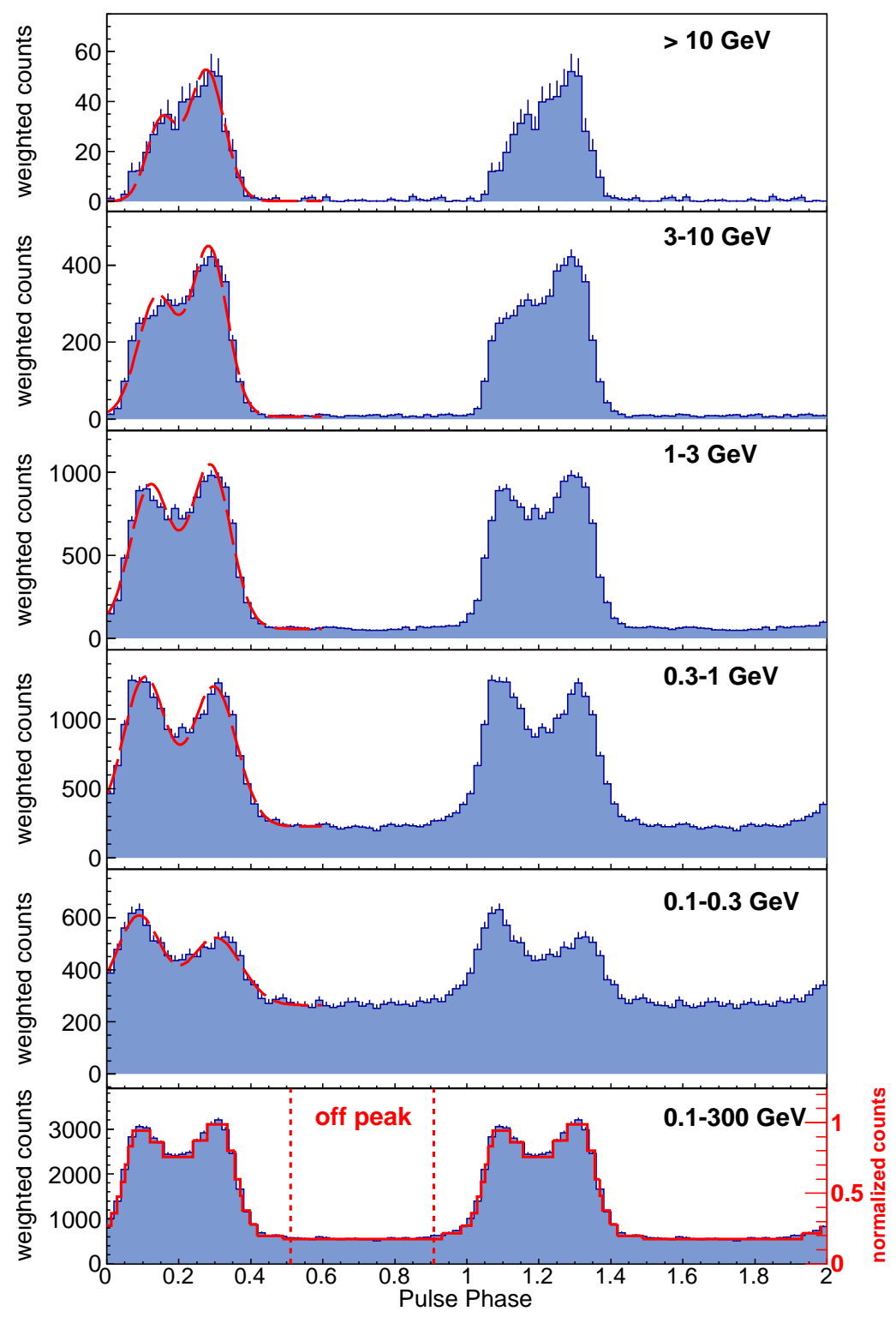

Fig. 2.- Weighted pulse profile of PSR J0007+7303 at different energies. Two rotational pulse periods are shown, with a resolution of 50 phase bins per period. The double Gaussian profile fitted to the light curves is shown with dashed red lines. The bottom panel shows the weighted pulse profile above $100 \mathrm{MeV}$. The Bayesian block decomposition is represented by red lines in the bottom panel. The region indicated by the red dashed lines is the off-peak phase. 
Table 1: Timing ephemeris of PSR J0007+7303.

\begin{tabular}{ll}
\hline \hline & \\
Parameter & Value \\
\hline \hline & \\
Pulsar name & PSR J0007+7303 \\
R.A. (J2000, Halpern et al. 2004) & $00: 07: 01.56$ \\
Decl. (J2000, Halpern et al. 2004) & $+73: 03: 08.1$ \\
MJD range & $54686.16-57370.50$ \\
Pulse frequency, $\nu\left(\mathrm{s}^{-1}\right)$ & $3.1658208(2)$ \\
First derivative of pulse frequency, $\dot{\nu}\left(\mathrm{s}^{-2}\right)$ & $-3.59(1) \times 10^{-12}$ \\
Second derivative of pulse frequency, $\ddot{\nu}\left(\mathrm{s}^{-3}\right)$ & $2.92 \times 10^{-22} \dagger$ \\
Epoch of frequency determination $(\mathrm{MJD})$ & 54952 \\
Glitch Epoch 1 (MJD) & 54952.92239 \\
Glitch Epoch 2 (MJD) & 55463.89923 \\
Glitch Epoch 3 (MJD) & 56369.56142 \\
& \\
\hline \hline
\end{tabular}

Note. — $\uparrow$ : model-predicted value assuming a braking index of 3 .
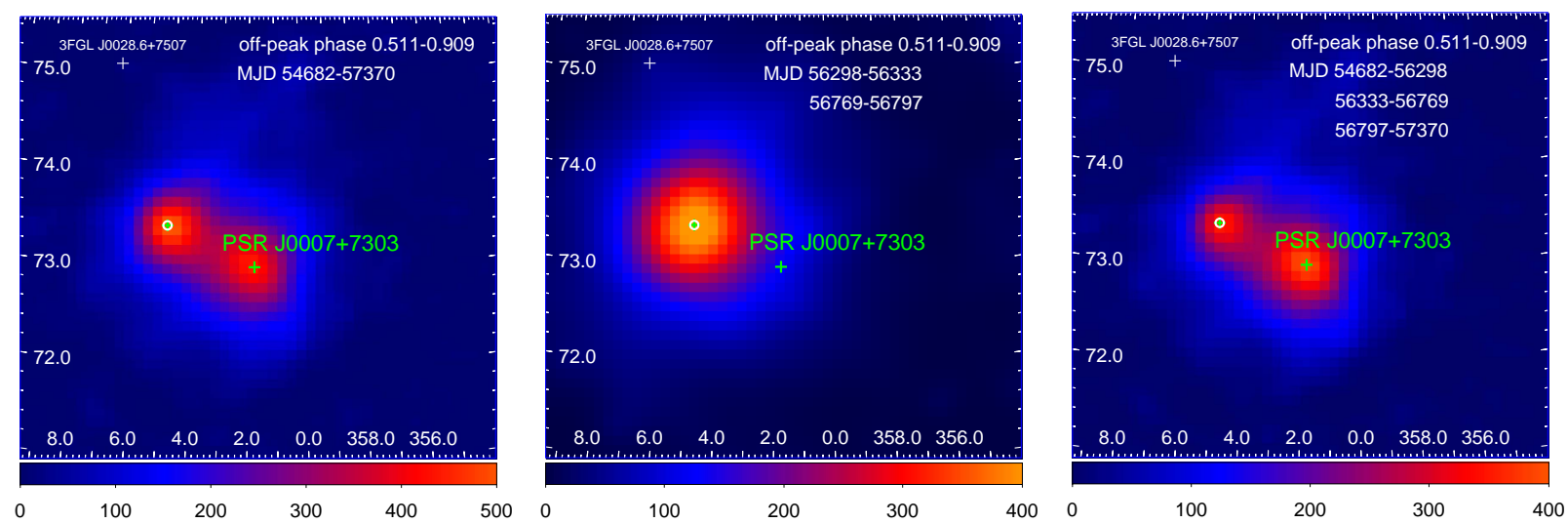

Fig. 3.- Left: TS maps of the Fermi-LAT field surrounding PSR J0007+7303 during the off-peak phase. The fitted position and $95 \%$ confidence error circle of a previously unknown gamma-ray source, Fermi J0020+7328, are shown with a green cross and a white circle. The $\mathrm{X}$-and Y-axis are RA and DEC referenced at J2000. Middle: the same as in the left panel, but only integrating the flare periods of Fermi J0020+7328. Right: the same as in the left panel, but integrating only the off-flare periods of Fermi J0020+7328. The off-peak phases and MJD ranges are shown in each panel. See text for details. 
Assuming a power law spectral shape, we produced the monthly binned long-term light curve of Fermi J0020+7328 (Figure 4, panel a \& b). The 95\% flux upper limits are calculated with Helene's method (Helene 1983) assuming a photon index of 2.0 if the TS value is below 9. Besides occasional flux fluctuations, two large flares are apparent, each lasting more than a month, which are indicated in Figure 4, panels a and b. A weekly light curve with an expanded scale of the two flares are shown in Figure 4, panel $\mathrm{c}-\mathrm{f}$. The flaring periods are MJD 56298 - 56333 (flare 1) and MJD 56769 - 56797 (flare 2), both of which are indicated in Figure 4, panel c - f. TS maps of the PSR J0007+7303 region during flaring and non-flaring periods are shown in Figure 3 .

The spectra of Fermi J0020+7328 in the different periods, both during the flares and in the off-flare period, are modeled by a power law and a power law with an exponential cutoff $\left(d N / d E=N_{0}\left(E / E_{0}\right)^{-\Gamma} \exp \left(-E / E_{0}\right) \mathrm{cm}^{-2} \mathrm{~s}^{-1} \mathrm{GeV}^{-1}\right)$. We compare the two models using the likelihood ratio test (Mattox et al. 1996). The $\Delta$ TS between the two models is less than 9, which indicates that a cutoff is not significantly preferred. The best-fit spectral parameters and corresponding TS values are listed in Table 2, while the spectral energy distributions (SEDs) are shown in Figure 5. The gamma-ray flux is $\sim 12$ times higher and the spectrum is harder during the flare period than outside it. We investigated the two flares individually. The flux levels of flares 1 and 2 are consistent within errors while the spectrum of flare 1 is softer than that of flare 2 (Table 2).

Active galactic nuclei (AGNs) are the dominant source population of the GeV sky (Ackermann et al. 2015). Since the gamma-ray flux of Fermi J0020+7328 is variable, it displays flares, and its spectrum is consistent with those of gamma-ray-detected AGNs (Ackermann et al. 2015), it is possible that this gamma-ray source is indeed an AGN.

Between 2006 - 2016 there have been seven observations of Fermi J0020+7328 with Swift. However, only two observations, 2006 November 4 (observation ID 00036187001) and 2009 September 11 (observation ID 00036187005), have sufficient Swift/XRT exposure (9.6 ks and $7.4 \mathrm{ks}$, respectively) for spectral analysis. The Swift/XRT counts map is shown in Figure 6, left panel. The quasar S50016+73 is the only X-ray source detected within the error circle of gamma-ray source Fermi J0020+7328 and is only 0:01 away, which argues for a possible association. S50016+73 is a Flat Spectrum Radio Quasar (FSRQ) with redshift of 1.781 (Lawrence et al. 1986). The Swift/XRT X-ray spectra of S50016+73 is well fit with a power law and the spectral parameters are shown in Table 3 . The X-ray photon index measured by Swift is consistent with a previous ROSAT result (Donato et al. 2001). Between the two Swift/XRT observations, there is also significant X-ray flux variability. However, because of the large uncertainty of the spectral index for the 2009 September 11 (observation ID 00036187005), we cannot claim a spectral change. With archival multi- 
wavelength data collected using the ASDC online services? we show the SED of S5 0016+73 including Swift/XRT data and the off-flare SED of Fermi J0020+7328 measured by FermiLAT in Figure 6, right panel. The off-flare SED of Fermi J0020+7328 is consistent with the overall SED of S5 0016+73. We propose that Fermi J0020+7328 is the GeV counterpart of S50016+73. Its gamma-ray photon index and flux level during the off-flare period are at the average of Fermi-LAT detected FSRQs (Ackermann et al. 2015). The flux level of S50016+73 during its flare period is in the upper end of gamma-ray-detected FSRQs. Considering its distance, the gamma-ray luminosity of S5 0016+73 is common among FermiLAT detected FSRQs.

S5 0016+73 is only $\sim 1$ degree away from PSR J0007+7303, and is more significant than the pulsar during off-peak phases (Figure 3, left panel). Taking the proximity and the size of the Fermi-LAT PSF into consideration, S5 0016+73 may affect the results obtained from PSR J0007+7303. To minimize its influence, we carried out the Fermi-LAT analysis of PSR J0007+7303 during the non-flare period of S50016+73 for both the off-peak and on-peak phases of PSR J0007+7303.

\subsection{PSR J0007+7303}

As mentioned, we carried out the off-peak analysis of PSR J0007+7303 during the non-flare period of S50016+73. The off-peak emission of PSR J0007+7303 was reported previously by Abdo et al. (2012) using two years of data with P6V11 IRFs and Abdo et al. (2013) using three years of data with P7V6 IRFs (P6V11 and P7V6 are both previous versions of LAT IRF ${ }^{7}$, yielding TS values of 40 and 71.5, respectively. However, no spectral cutoff was detected in these analyses, probably due to the limited statistics. To explore the

\footnotetext{
6 http://tools.asdc.asi.it/SED/

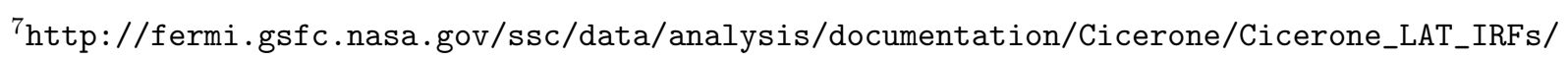
IRF_overview.html
} 

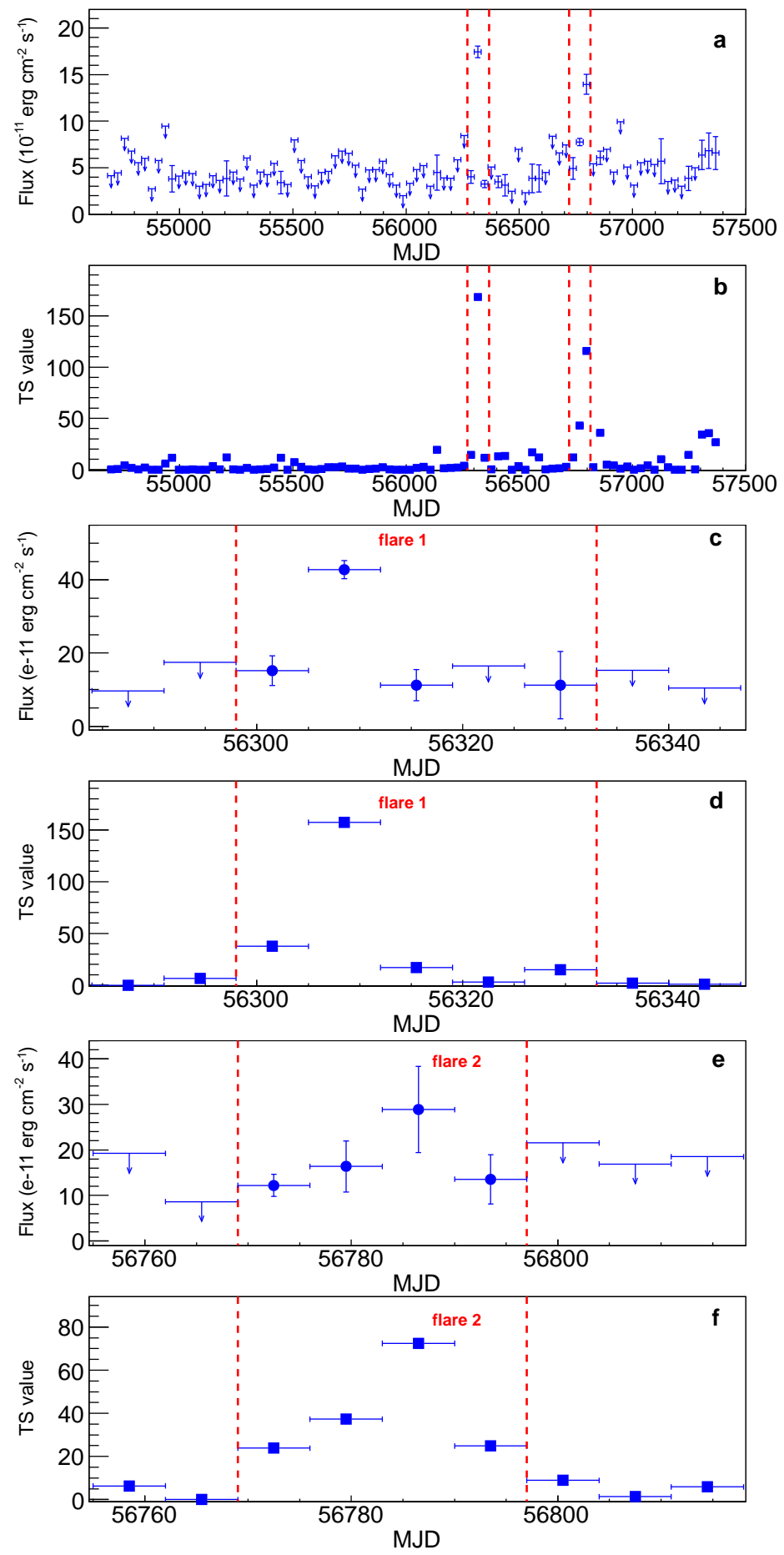

Fig. 4.- Panels a \& b: Monthly gamma-ray flux and TS value of Fermi J0020+7328. Two possible flares are indicated with red dashed lines. Panels c \& d: Expanded scale, weekly gamma-ray flux and TS value evolution around flare 1. Panels e \& f: Expanded scale, weekly gamma-ray flux and TS value evolution around flare 2. The periods of the two flares defined in the text are indicated with red dashed lines in panels $\mathrm{c}-\mathrm{f}$. 
Table 2: Fermi-LAT fitted spectral parameters of Fermi J0020+7328 during the off-peak phase of PSR J0007+7303.

\begin{tabular}{llll}
\hline \hline Time Interval & Spectral Index & TS & $\begin{array}{l}\text { Energy Flux, } 0.1-300 \mathrm{GeV} \\
10^{-11} \mathrm{erg} \mathrm{cm}^{-2} \mathrm{~s}^{-1}\end{array}$ \\
\hline \hline & & & \\
Flaring intervals combined & $2.36 \pm 0.01 \pm 0.04$ & 357 & $16.7 \pm 0.8 \pm 0.3$ \\
Flare 1 (MJD 56298-56333) & $2.43 \pm 0.02 \pm 0.03$ & 197 & $16.4 \pm 0.8 \pm 0.5$ \\
Flare 2 (MJD 56769-56797) & $2.22 \pm 0.12 \pm 0.07$ & 134 & $16.6 \pm 3.2 \pm 1.5$ \\
& & & \\
\hline & $2.54 \pm 0.06 \pm 0.16$ & 201 & $1.4 \pm 0.2 \pm 0.1$ \\
Non-flaring intervals & & \\
\hline \hline
\end{tabular}

Note. - The first (second) uncertainties correspond to statistical (systematic) errors.
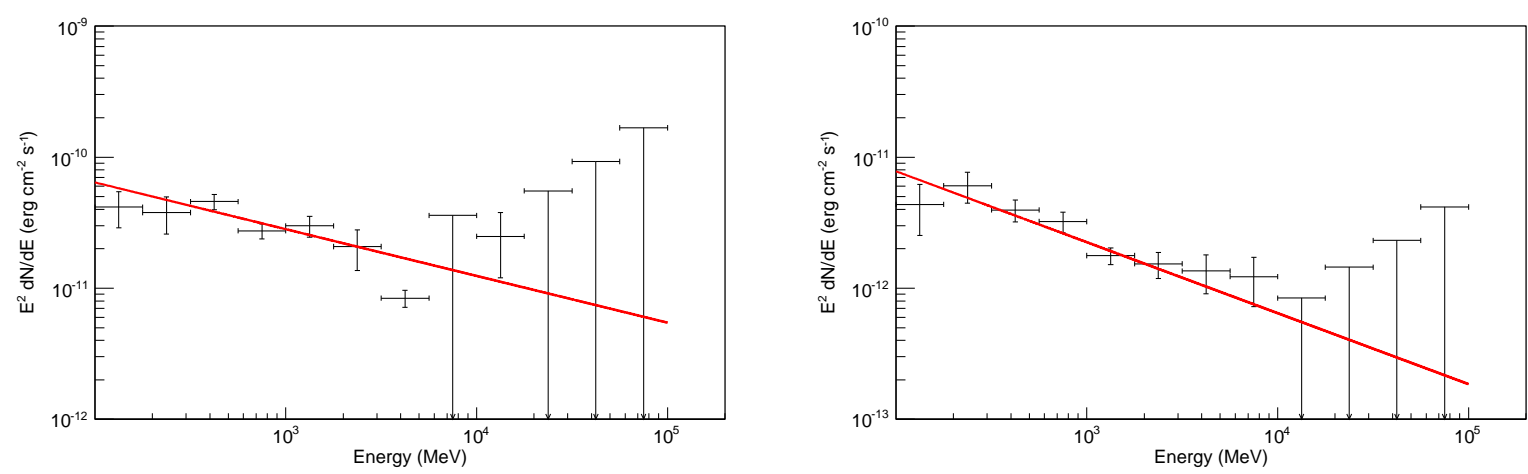

Fig. 5.- Fermi-LAT spectra of the gamma-ray source Fermi J0020+7328 during flare (left) and non-flare (right) periods. The gtlike fitted models are shown with red lines.

Table 3: Swift/XRT fitted spectral parameters of S5 0016+73.

\begin{tabular}{cccccc}
\hline \hline Observation ID & Date & Spectral Index & $\begin{array}{c}\text { Flux, } 0.3-10 \mathrm{keV} \\
10^{-12} \mathrm{erg} \mathrm{cm}^{-2} \mathrm{~s}^{-1}\end{array}$ & $\begin{array}{c}\mathrm{N}_{H} \\
10^{22} \mathrm{~cm}^{-2}\end{array}$ & Reduced $\chi^{2} /$ (D.O.F.) \\
\hline \hline & & & & & \\
00036187001 & 2006 November 4 & $1.51 \pm 0.16$ & $4.1 \pm 0.3$ & $0.18 \pm 0.07$ & $0.59(21)$ \\
00036187005 & 2009 September 11 & $1.81_{-0.41}^{+0.44}$ & $1.4_{-0.2}^{+0.5}$ & $0.31_{-0.27}^{+0.30}$ & $1.38(3)$ \\
\hline \hline
\end{tabular}



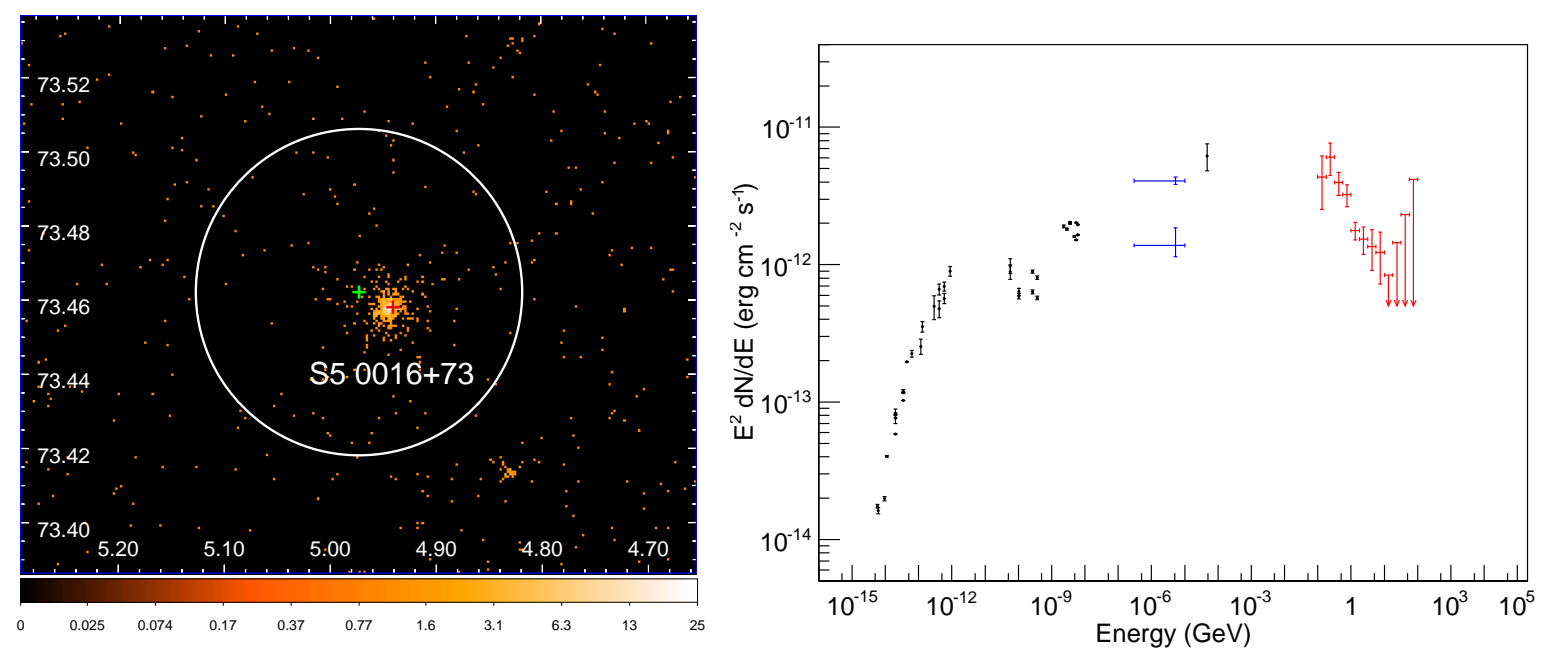

Fig. 6. - Left: Swift/XRT counts map from 2006 November 4 observation of the previously unknown gamma-ray source, Fermi J0020+7328, in the $0.3-10 \mathrm{keV}$ range. The Fermi-LAT best-fit position and 95\% confidence error circle are shown with a green cross and a white circle respectively. The position of $\mathrm{S} 50016+73$ is indicated with a red cross. The X- and Y-axes are RA and DEC referenced at J2000. Right: Non-simultaneous SED of S5 0016+73. The Swift/XRT data are shown in blue while the off-flare SED of Fermi J0020+7328 is shown in red. 
possible cutoff in the off-peak spectrum, we modeled PSR J0007+7303 using a power law function with and without an exponential cutoff. We compare the two models using the likelihood ratio test, leading to a $\Delta \mathrm{TS}$ of 12.4 , which indicates that the significance of the spectral cutoff is $\sim 3.5 \sigma$. PSR J0007+7303 is detected with a TS value of 262 and the fitted parameters are shown in Table 4. The SED is shown in Figure 7. This is the first hint of the existence of a spectral cutoff during the off-peak phase of PSR J0007+7303.

Abdo et al. (2012) excluded a point-like hypotheses for the off-pulse emission at the $95 \%$ level and thus reported a marginal detection of extent while in the $2 \mathrm{PC}$ the $\mathrm{TS}_{\text {ext }}$ was calculated to be $10.8(\sim 3.3 \sigma)$. With the use of more advanced response and diffuse models, significantly more data accumulated, and the newly detected gamma-ray source Fermi J0020+7328 included in the model, we performed the likelihood analysis again to check for the possible extension. In the off-peak gamma-ray emission, we fitted an extended disk to PSR J0007+7303 using Pointlike, yielding a $\mathrm{TS}_{\text {ext }}=1.3$; the disk is not favored. The off-peak gamma-ray emission of PSR J0007+7303 is not extended.

As a check, adopting only the two years of data analyzed by Abdo et al. (2012), we fitted an extended disk to PSR J0007+7303 during the off-peak phases using Pointlike with Fermi J0020+7328 included in the model, leading to $\mathrm{TS}_{\text {ext }}=2.3$ (87\% confidence level), which is consistent with the $95 \%$ confidence level measured by Abdo et al. (2012) and does not imply a significantly extended emission either. For a further check, we excluded Fermi $\mathrm{J} 0020+7328$ from the model and repeated the above analysis. It leads to a disk of radius $0.62 \pm 0^{\circ} .13$ located at $\mathrm{RA}_{J 2000}=1.847$ and $\mathrm{Dec}_{J 2000}=73.219$ and a $\mathrm{TS}_{\text {ext }}=9.4(99.9 \%$ confidence level). These values are consistent with the $0.7 \pm 0.3$ extension at $95 \%$ confidence level reported in Abdo et al. (2012). Thus, considering both the hint of the spectral cutoff at more than $3 \sigma$ and the point-like morphology, we propose that the off-peak gamma-ray emission of PSR J0007+7303 originates from the magnetosphere of the pulsar rather than the PWN or the SNR CTA 1.

\section{On-peak analysis}

For the on-peak analysis, the normalizations of all 3FGL sources within three degrees of PSR J0007+7303 were left free in the model. For Fermi J0020+7328 and all the 3FGL sources beyond three degrees, the normalizations adopted were that of the off-peak fitted values rescaled to the ratio of the width of the on-peak to off-peak phase intervals and then fixed. All other spectral parameters were fixed at off-peak fitted value except for PSR J0007+7303. 

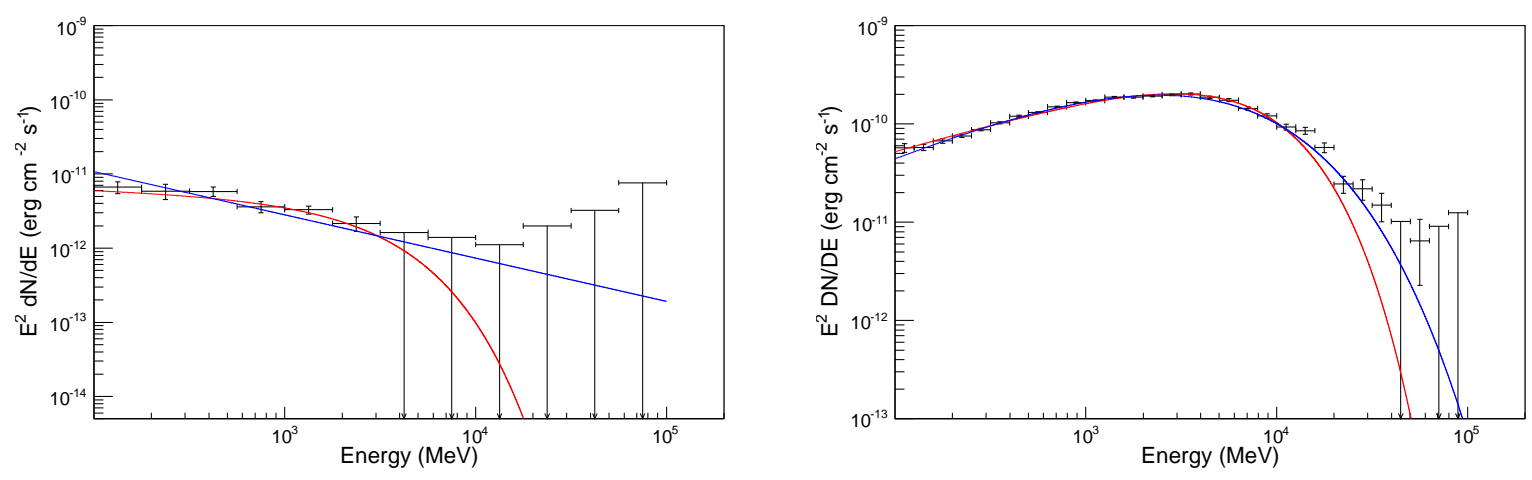

Fig. 7.- Fermi-LAT spectra of PSR J0007+7303 during off-peak (left) and on-peak (right) phases. Maximum likelihood models fitted with gtlike are shown with red lines (power law with exponential cutoff) and blue lines (power law, left and power law with sub-exponential cutoff, right).

Table 4: Fermi-LAT spectral parameters of PSR J0007+7303 during off-peak and on-peak phases.

\begin{tabular}{cccccc}
\hline Phase Interval & Spectral Index & $\begin{array}{c}\text { Cutoff Energy } \\
(\mathrm{GeV})\end{array}$ & $b$ & TS & $\begin{array}{c}\text { Flux, } 0.1-300 \mathrm{GeV} \\
10^{-11} \mathrm{erg} \mathrm{cm}^{-2} \mathrm{~s}^{-1}\end{array}$ \\
\hline \hline & & & & & \\
off-peak & $2.09 \pm 0.21 \pm 0.83$ & $2.7 \pm 1.2 \pm 1.3$ & - & 262 & $1.5 \pm 0.2 \pm 0.1$ \\
on-peak & $1.44 \pm 0.01 \pm 0.03$ & $5.2 \pm 0.1 \pm 0.4$ & - & 190317 & $68.3 \pm 0.5 \pm 0.4$ \\
& $1.13 \pm 0.06 \pm 0.15$ & $1.14 \pm 0.36 \pm 0.50$ & $0.57 \pm 0.04 \pm 0.06$ & 190278 & $68.1 \pm 0.5 \pm 1.9$ \\
& & & & & \\
\hline \hline
\end{tabular}

Note. - The first (second) uncertainties correspond to statistical (systematic) errors. 
In modeling the on-peak phase of PSR J0007+7303, we have first considered a power law with an exponential cutoff. The best-fit parameters are shown in Table 4.

The SED of the on-peak phase is shown in Figure 7 and the fitted power law with an exponential cutoff spectral shape is shown with a red line. At higher energies, the SED points deviate from the fitted spectral shape. We studied alternative spectral shapes, specifically a power law with a sub-exponential cutoff $\left(d N / d E=N_{0}\left(E / E_{0}\right)^{-\Gamma} \exp \left(-E / E_{0}\right)^{b} \mathrm{~cm}^{-2} \mathrm{~s}^{-1}\right.$ $\mathrm{GeV}^{-1}$, leaving the exponential index $b$ free). The best-fit parameters are shown in Table 4 . The parameter $b$ is found to be $0.57 \pm 0.04 \pm 0.06$ (the first/second uncertainties correspond to statistical/systematic errors.), which is consistent with the $b$ value for young pulsars derived by a stacking analysis (McCann 2015). Using the likelihood ratio test, the $\Delta$ TS between the two models of the region is 125 , which indicates that the significance of the sub-exponential cutoff is $\sim 11 \sigma$. The fitted power law with sub-exponential cutoff spectral shape is shown with a blue line in Figure 7, and it models the SED well.

As previously reported for the Vela and Geminga pulsars (Abdo et al. 2010, Bonnefoy et al. 2015), a value of $b<1$ could be interpreted as a blend of different phase-resolved $b=1$ spectra having different cutoff energies. To explore this possibility, we carried out a phaseresolved spectral analysis. The best-fit model from the on-peak phase-averaged fit is used as the input model for the phase-resolved fit: i.e., for this analysis, all parameters except those associated with the pulsar were fixed to the value derived from the on-peak phase fit. In each phase range, the pulsar spectrum is modeled as both a power law with a simple exponential cutoff and with a sub-exponential cutoff. Figure 8 shows the phase-resolved spectral parameters of PSR J0007+7303 in different phase bins. From the phase-resolved fits, we note that the $b$ parameter is consistently lower than 1 in all tested phase bins. We further note that for each phase bin, the significance of the sub-exponential cutoff is greater than $3 \sigma$. Thus, for the first time we have shown that both the on-peak averaged spectrum as well as the phase-resolved spectra of PSR J0007+7303 are better described by a power law with a sub-exponential cutoff function.

\section{Light curve and spectral variability analysis}

To check for long-term flux variability of PSR J0007+7303 we performed a phase averaged likelihood analysis similar to that done in Section 5 . We selected the length of 
the time bin as 60 days and used the same model as described in Section 5 but rescaled to the overall pulse phase. PSR J0007+7303 is modeled as a power law with an exponential cutoff instead of a sub-exponential cutoff because of the lower statistics in each time bin. Figure 9, top panel shows the resulting long-term light curve of PSR J0007+7303, which is well fitted by a constant line, yielding a reduced $\chi^{2}$ of 1.36 . No significant flux variation is detected. Three glitches have been detected from PSR J0007+7303 (Table 1) and no flux variations are detected in the long-term light curve around the glitches. We have checked for any changes in the spectrum of the pulsar around the glitches. To accomplish this, the Fermi-LAT data were split into four bins around the glitches. Adopting the power law with exponential cutoff model, we performed a likelihood analysis for PSR J0007+7303 in these four time bins. The flux and spectral parameters are shown in Figure 9 and are similar in the four epochs. No change in the integral flux above $100 \mathrm{MeV}$ is seen.

Adopting the best-fit spatial and spectral model derived from the above phase averaged analysis, we calculated the probabilities of photons coming from PSR J0007+7303 within a radius of $3^{\circ}$ using gtsrcprob and produced a weighted pulsed light curve based on them. The bottom panel of Figure 2 shows the folded, pulsed light curve above $100 \mathrm{MeV}$. The remaining panels of the same figure show the light curve in narrower energy bands. The light curve shows two distinct peaks, which is consistent with the profile reported by Abdo et al. (2012) and the 2PC. To locate the two peaks, we fitted the light curve with a double Gaussian profile (Figure 2). The first (P1) and second (P2) peaks are at $\phi=0.113 \pm 0.001$ and $\phi=0.293 \pm 0.001$, respectively. The separation between the means of the two peaks is $0.180 \pm 0.002$. The widths of $\mathrm{P} 1$ and $\mathrm{P} 2$ evolve with energy, leading to narrower peaks at higher energies (Figure 10, top and middle). A similar evolution was also observed in Geminga (Abdo et al. 2010). The relative strength of P1 and P2 decreases significantly from low to high energies (Figure 10, bottom panel), which again is consistent with what was first reported by Abdo et al. (2012).

\section{DISCUSSION}

Using more than seven years of Fermi-LAT data and a contemporaneous ephemeris, we carried out a detailed analysis of PSR J0007+7303 during its off-peak and its on-peak phase intervals.

During the off-peak phase, PSR J0007+7303 is significantly detected with a TS value of 262. An exponential cutoff at $2.7 \pm 1.2 \pm 1.3 \mathrm{GeV}$ is tentatively detected in its spectrum, 
with a significance of $3.5 \sigma$. We explored the possible extension of PSR J0007+7303 during the off-peak phase, but a point-like source is favored $\left(\mathrm{TS}_{e x t}=1.3\right)$. The point-like nature of the emission together with the potential cutoff at $\mathrm{GeV}$ energies argue for a magnetospheric origin of the off-peak gamma-ray emission of PSR J0007+7303.

Neither a point-like source nor extended gamma-ray emission was detected from PSR J0007+7303 between 10 and $300 \mathrm{GeV}$ during the off-peak phase. By removing the point source model of PSR J0007+7303, assuming the same position and the 0.3-degree extension detected by VERITAS (Aliu et al. 2013), we calculated an upper limit for the possible emission coming from the PWN or the SNR CTA 1, of $6.5 \times 10^{-12} \mathrm{erg} \mathrm{cm}^{-2} \mathrm{~s}^{-1}$ at 99\% confidence level, with Helene's method (Helene 1983) assuming a photon index of 2.0 and considering the systematics $(10-300 \mathrm{GeV})$. In the case of the highest energies, the $\mathrm{TeV}$ emission detected by VERITAS is most likely coming from the PWN. The molecular mass in the vicinity of the complex is not enough to explain the $\mathrm{TeV}$ emission even under favorable assumptions for the cosmic-ray acceleration properties of the SNR (see the discussion by Martin et al. 2016). The new upper limit we impose on the GeV emission from the PWN is not in conflict with detailed multi-frequency models (Aliu et al. 2013, Torres et al. 2014). This PWN remains, however, a difficult case: it is unique in requiring a relatively high magnetization (as compared with other PWNe detected). The latter and the SNR estimated age may indicate that the nebula (or at least part of it) is already contracting. However, even considering that the PWN could already have passed reverberation, the needed magnetization is still high (Matin, Torres, \& Pedaletti 2016).

Off-peak emission of 26 young pulsars and 8 millisecond pulsars has been significantly detected (2PC). Their off-peak luminosities range from $\sim 10^{32}$ to $\sim 10^{35} \mathrm{erg} \mathrm{\textrm {s } ^ { - 1 }}$ and PSR J0007+7303 is near the geometric average $\left(\mathrm{L}_{\text {off peak }}=3.5 \times 10^{33} \mathrm{erg} \mathrm{s}^{-1}\right)$. Considering a distance of $1.4 \mathrm{kpc}$ and a spin-down power $\dot{E}=-I \Omega \dot{\Omega}$ ( $I$ is the pulsar's moment of inertia $10^{45} \mathrm{~g} \mathrm{~cm}^{2}, \Omega$ and $\dot{\Omega}$ are the pulsar spin frequency and the first derivative of spin frequency) of $4.5 \times 10^{35} \mathrm{erg} \mathrm{s}^{-1}$, the off-peak emission efficiency $\left(\mathrm{L}_{\text {off peak }} / \dot{E}\right)$ of PSR J0007+7303 is $0.8 \%$, which is among the lowest of pulsars with magnetospheric off-peak emission (2PC, Figure 14). The on-peak emission efficiency $\left(\mathrm{L}_{\text {on peak }} / \dot{E}\right)$ of PSR J0007+7303 is $\sim 36.5 \%$.

For the on-peak phase, PSR J0007+7303 could be modeled by a power law with a subexponential cutoff, which is favored over an exponential cutoff with a significance above $11 \sigma$ for the phase-averaged spectrum (Table 4) and of $3 \sigma$ for the phase-resolved spectra (Figure 8). This makes PSR J0007+7303 the fourth pulsar having an established sub-exponential cutoff spectrum in at least some phase range, besides Geminga, Vela, and Crab (see e.g., 2PC; Bochenek \& McCann 2015). PSR J0007+7303 showed a two-peak pulse profile. The ratio of P1 and P2 evolves significantly with energy (Figure 10). At low energies, the strengths of 
$\mathrm{P} 1$ and P2 are comparable, yet P2 is more prominent at higher energies (Figure 2), similar to the tendency observed in the Crab, Vela, and Geminga pulsars (Kanbach 1999; Aleksić et al. 2014). This is consistent with the lower cut-off energy of P1 than that obtained for P2 (Figure 8, left panel).

Several hypotheses have been proposed to explain the deviation of the spectral cutoff from a pure exponential one. In the outer-gap model of pulsar radiation, the high energy emission originates at high altitudes from the neutron star (see e.g., Cheng et al. 1986a, Cheng et al. 1986b, Romani 1996). A spectral shape represented by a power law with an exponential cut-off is expected (see e.g., Prosekin et al. 2013, Viganò et al. 2015a,b). In these kinds of models, the accelerating electric field depends on the height in the gap (Hirotani 2006, Hirotani 2015). Particles at distinct heights will be accelerated to different energies, leading to a range of cut-off energies. The appearance of a sub-exponential cutoff could be taken as evidence that the emission of different pulsar phases is produced by different particle acceleration zones (or via different processes) with different radiation-reaction energies. As a result of the wide emission beams in the outer-gap, emission at a particular phase are a combination of different beams and different cutoff energies. Therefore a blend of different cutoff energies could plausibly lead to the sub-exponential spectra. Leung et al. (2014) also proposed that the accelerating voltage in a given gap is unstable. Emission from even a single emitting zone is a convergence of various accelerate states, which will lead to the sub-exponential cutoff in a pulsar spactra. Such sub-exponential cutoffs can also be due to the contribution of a second component, arising from inverse Compton emission of electrons upscattering off soft photon fields (Hirotani 2015; Lyutikov 2013). However, we note that the physical interpretation of the meaning of $b<1$ should be considered as provisional, since it may simply depend on our sensitivity. With increased statistics we have seen that values of $b<1$ are needed to fit first the phase-averaged spectrum, then the phase-resolved ones. It may well be that even in the smaller phase bins considered we are summing up contributions having different acceleration features and thus producing sub-exponential cutoffs as a result of this sum. By reducing even further the phase bins, we would come to a situation in which cutoff power laws with $b=1$ and with $b<1$ would not produce significantly different fits. Up to what extent the existence of $b<1$ is physical and not a problem of sensitivity (too large phase bins for the level of statistics attained) is still a subject of controversy. For a phase-averaged analysis, we found no flux variability in the long-term light curve. The integrated flux level and spectral parameters are consistent during all epochs preceding and following the glitches.

We have identified Fermi J0020+7328, a previously unknown, flaring gamma-ray source appearing (due to the relative strength of both sources) only during the off peak phases of PSR J0007+7303. The most probable counterpart for this source is S5 0016+73. 
The Fermi LAT Collaboration acknowledges generous ongoing support from a number of agencies and institutes that have supported both the development and the operation of the LAT as well as scientific data analysis. These include the National Aeronautics and Space Administration and the Department of Energy in the United States, the Commissariat à l'Energie Atomique and the Centre National de la Recherche Scientifique / Institut

National de Physique Nucléaire et de Physique des Particules in France, the Agenzia Spaziale Italiana and the Istituto Nazionale di Fisica Nucleare in Italy, the Ministry of Education, Culture, Sports, Science and Technology (MEXT), High Energy Accelerator Research Organization (KEK) and Japan Aerospace Exploration Agency (JAXA) in Japan, and the K. A. Wallenberg Foundation, the Swedish Research Council and the Swedish National Space Board in Sweden.

Additional support for science analysis during the operations phase is gratefully acknowledged from the Istituto Nazionale di Astrofisica in Italy and the Centre National d'Études Spatiales in France.

We acknowledge the assistance from Dr. M. Kerr with the gamma-ray ephemeris for PSR J0007+7303, Dr. M. Razzano and P. Saz Parkinson for discussions. We acknowledge the support from the grants AYA2015-71042-P, SGR 2014-1073 and the National Natural Science Foundation of China via NSFC-11473027, NSFC-11503078, NSFC-11133002, NSFC11103020, XTP project XDA 04060604 and the Strategic Priority Research Program "The Emergence of Cosmological Structures" of the Chinese Academy of Sciences, Grant No. XDB09000000.

\section{REFERENCES}

Abdo, A. A., Ackermann, M., Atwood, W. B., et al. 2008, Sci., 322, 1218

Abdo, A. A., Ackermann, M., Ajello, M., et al. 2010, ApJ, 712, 1209

Abdo, A. A., Ackermann, M., Ajello, M., et al. 2010, ApJ, 713, 154

Abdo, A. A., Wood, K. S., DeCesar, M. E., et al. 2012, ApJ, 744, 146

Abdo, A. A., Ajello, M., Allafort, A., et al. 2013, ApJS, 208, 17 (2PC)

Acero, F., Ackermann, M., Ajello, M., et al. 2015, ApJS, 218, 23 (3FGL)

Acero, F., Ackermann, M., Ajello, M., et al. 2016, ApJS, 223, 26

Ackermann, M., Ajello, M., Allafort, A., et al. 2012, ApJS, 203, 4 
Ackermann, M., Ajello, M., Atwood, W. B., et al. 2015, ApJ, 810, 14

Aleksić, J., Ansoldi, S., Antonelli, L. A., et al. 2014, A\&A, 565, 12

Aliu, E., Archambault, S., Arlen, T., et al. 2013, ApJ, 764, 38

Atwood, W. B., Abdo, A. A., Ackermann, M., et al. 2009, ApJ, 697, 1071

Bertsch D. L., et al. 1992, Nature, 357, 306

Bonnefoy, S., Brazier, K. T. S., Fichtel, C. E., et al. 2015, PoS, ICRC2015, 738

Bochenek C., \& McCann A., 2015, proceeding of science of the 34th ICRC, arXiv:1507.03136

Burrows D. N., Hill J. E., Nousek J. A., et al. 2005, Space Sci. Rev., 120, 165

Caraveo, P. A., De Luca, A., Marelli, M., et al. 2010, ApJ, 725, L6

Cheng, K. S., Ho, C., \& Ruderman, M. 1986a, ApJ, 300, 500

Cheng, K. S., Ho, C., \& Ruderman, M. 1986b, ApJ, 300, 522

de Jager, O. C., \& Büsching, I. 2010, A\&A, 517, L9

de Jager, O. C., Raubenheimer, B. C., \& Swanepoel, J. W. H. 1989, A\&A, 221, 180

Donato, D., Ghisellini, G., Tagliaferri, G.,\& Fossati, G., 2001, A\&A, 375, 739

Halpern, J. P., Gotthelf, E. V., Camilo, F., Helfand, D. J., \& Ransom, S. M. 2004, ApJ, 612, 398

Halpern, J. P., Camilo, C., \& Gotthelf, E. V. 2007, ApJ, 668, 1154

Harris, D. E., \& Roberts, J. A. 1960, PASP, 72, 347

Helene, O., 1983, NIMPR, 212, 319

Hirotani, K. 2006, Modern Physics Letters A, 21, 1319

Hirotani, K. 2015, ApJ, 798, L40

Hobbs, G., Edwards, R., \& Manchester, R. 2006, Chin. J. Astron. Astrophys. Suppl., 6, 189

Jackson, B., Scargle, J. D., Barnes, D., et al. 2005, ISPL, 12, 105

Kanbach, G., 1999, ApL \& C, 38, 17 
Kerr, M. 2011, PhD thesis, Univ. Washington

Kerr, M., Ray, P. S., Johnston, S., Shannon, R. M., Camilo, F., 2015, ApJ, 814, 128

Lande, J., Ackermann, M., Allafort, A., et al., 2010, ApJ, 756, 5

Lawrence, C. R., Pearson, T. J., Readhead, A. C. S., Unwin, S. C., 1986, AJ, 91, 494

Leung,G. C. K, Takata, J., Ng, C. W., et al. 2015, ApJ, 797, 13

Lin, L. C. C., Huang, R. H. H., Takata, J., et al. 2010, ApJ, 725, L1

Lin, L. C. C., Hui, C. Y., Li, K. T., et al. 2014, ApJ, 793, 8

Lyutikov, M., 2013, MNRAS, 431, 2580

Mattox J. R., Bertsch, D. L., Chiang, J., et al., 1996, ApJ, 461, 396

Martin J., Torres D. F., Pedaletti G. 2016, MNRAS, in press (astro-ph/1603.09328)

McCann, A. 2015, ApJ, 804, 86

Mignani, R. P., de Luca, A., Rea, N., et al. 2013 MNRAS, 430, 1354

Pineault, S., Landecker, T. L., Madore, B., \& Gaumont-Guay, S. 1993, AJ, 105, 1060

Pineault, S., Landecker, T. L., Swerdlyk, C. M., \& Reich, W. 1997, A\&A, 324, 1152

Prosekin, A. Y., Kelner, S. R., \& Aharonian, F. A. 2013, arXiv: 1305.0783

Ray, P. S., Kerr, M., Parent, D., et al. 2011, ApJS, 194, 17

Romani, R. W., 1996, ApJ, 470, 469

Sambruna, Rita M., 1997, ApJ, 487, 536

Seward, F. D., Schmidt, B., \& Slane, P. 1995, ApJ, 453, 284

Scargle, J. D., Norris, J. P., Jackson, B., \& Chiang, J. 2013, ApJ, 764, 167

Slane, P., Seward, F. D., Bandiera, R., et al. 1997, ApJ, 485, 221

Slane, P., Zimmerman, E. R., Hughes, J. P., et al. 2004, ApJ, 601, 1045

Torres, D. F., Cillis, A., Martín, J., \& de Ona Wilhelmi, E. 2014, JHEAp 1, 31

Viganò, D., \& Torres, D. F., 2015a, MNRAS, 449, 3755 
Viganò, D., Torres, D. F., \& Martín, J. 2015b, MNRAS, 453, 2599 

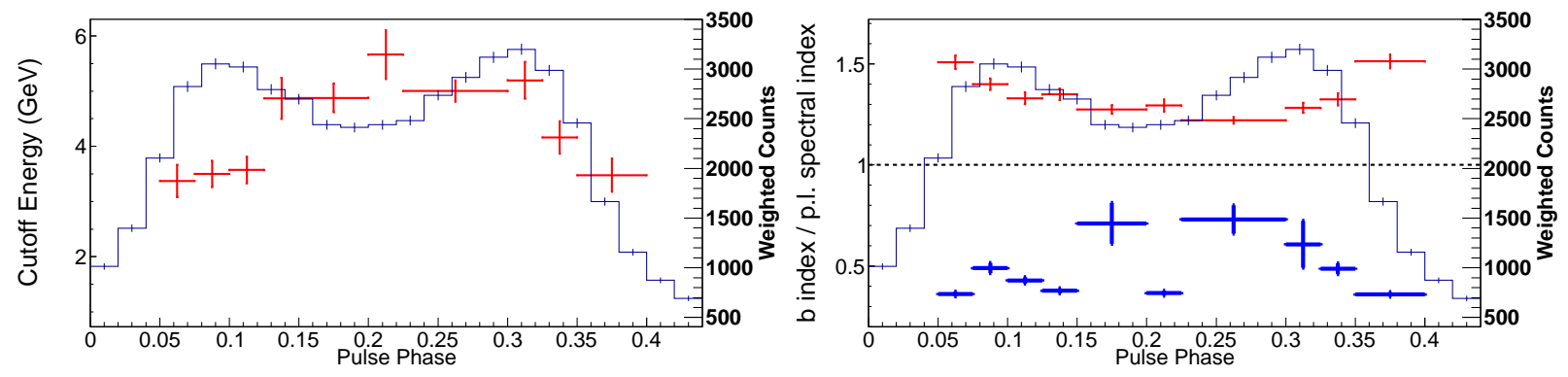

Fig. 8. - The phase-resolved spectral parameters of PSR J0007+7303. The histogram in each panel shows the weighted phaseogram of PSR J0007+7303 between 0.1 and $300 \mathrm{GeV}$ (same as Figure 2, bottom panel). The range in pulsar phase is restricted to $0.0-0.44$. Red points in the left and right panels correspond to the cutoff energy and the spectral index of the power law with exponential cutoff model, respectively. The blue points in the right panel show the values of $b$ in the model of a power law with sub-exponential cutoff. The dotted horizontal line indicates an index value of unity. 

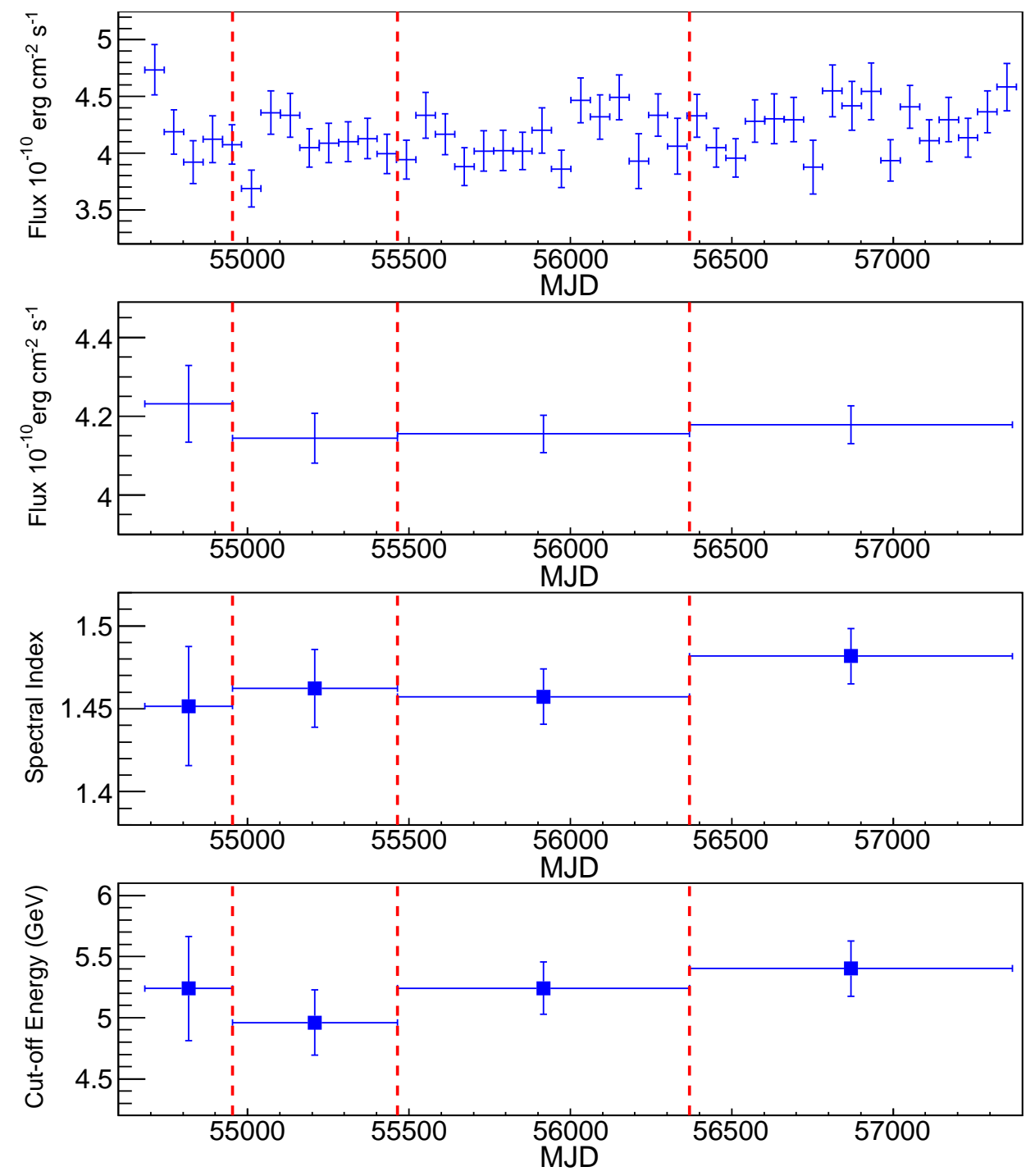

Fig. 9.- From top to bottom: flux of PSR J0007+7303 in 0.1-300 GeV as a function of time in 60-day time bins; integrated flux, spectral index and cut-off energy of PSR J0007+7303 in 0.1-300 GeV for epochs separated by the glitches. The dashed vertical red lines mark the time of glitches. 

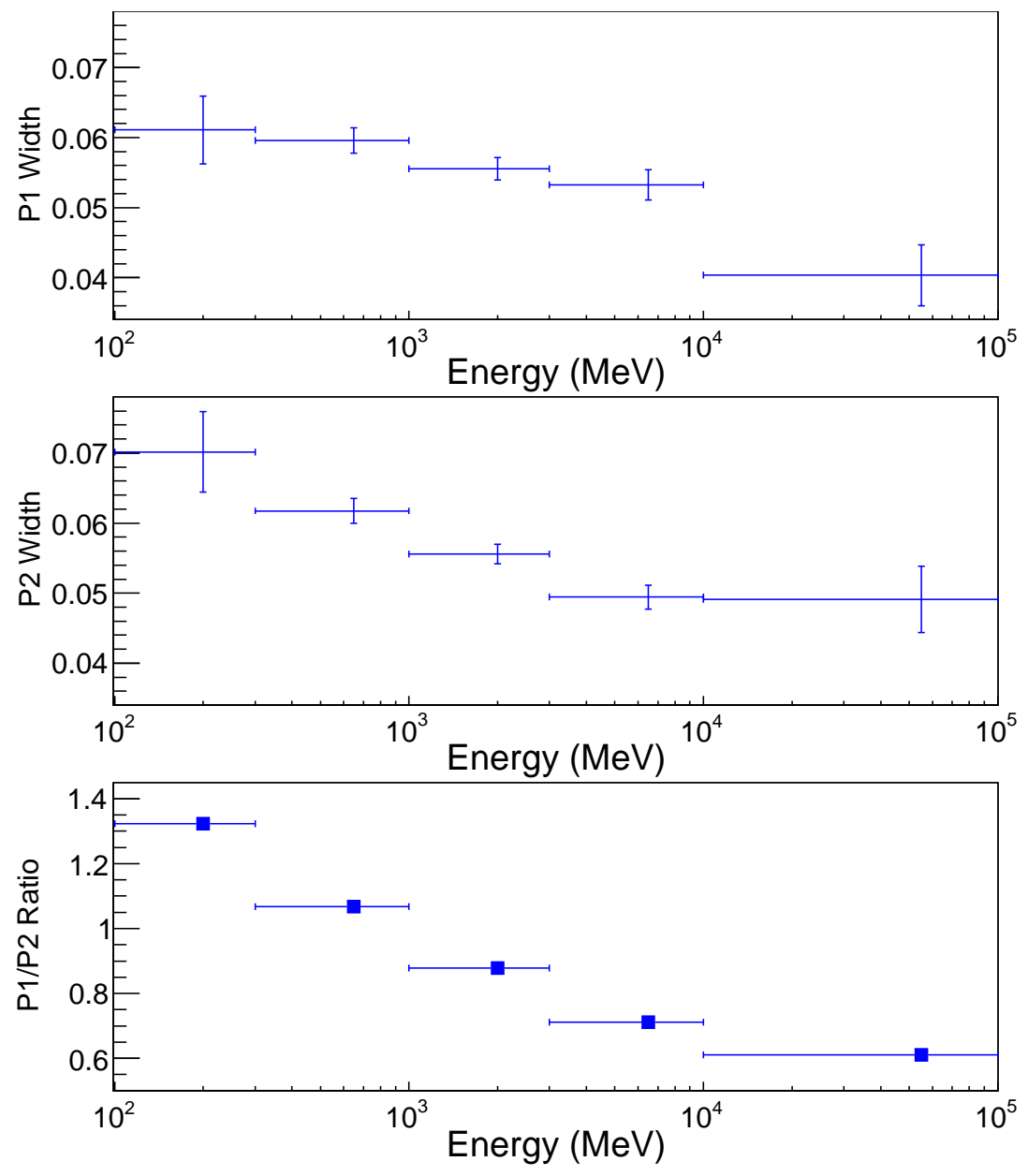

Fig. 10.- From top to bottom, energy evolution of the width of P1, the width of P2, and the $\mathrm{P} 1 / \mathrm{P} 2$ ratio. The energy bins are the same as in Figure 2 . 